\title{
Zur Geschichte der Kühlkost und des Tiefgefrierens
}

\author{
Teuteberg, Hans Jürgen
}

First published in:

Zeitschrift für Unternehmensgeschichte, 36. Jg., Heft 3, S. 139 - 155, Stuttgart 1991

Münstersches Informations- und Archivsystem multimedialer Inhalte (MIAMI) URN: urn:nbn:de:hbz:6-06429505868 


\section{Zur Geschichte der Kühlkost und des Tiefgefrierens}

Die Technik des Tiefgefrierens von Lebensmitteln gehört zu den großen bleibenden Innovationen des modernen Industriezeitalters, da durch sie eine jahrtausendealte Vorratswirtschaft entscheidend erweitert und die damit verbundene Nahrungskultur revolutionär mitumgestaltet wurde. Um so merkwürdiger mutet es an, $\mathrm{da} B$ es in Deutschland immer noch an einer wissenschaftlichen Gesamtdarstellung über die Entwicklung der Tiefkühlwirtschaft fehlt, obwohl sich gerade deutsche Erfinder und Unternehmer auf diesem Gebiet auch international als führend hervortaten. ${ }^{1}$ Vom Standpunkt der Geschichtswissenschaft erscheint es daher dringend erforderlich, die zerstreuten Abhandlungen und Notizen, die nur sachlich, räumlich oder zeitlich begrenzte Einzelprobleme unvollkommen beleuchten, durch eine systematische Ausschöpfung des zeitgenössischen Quellenmaterials ergänzend zu überprüfen und nach allgemeinen Erklärungen zu suchen, die dann in den Gang der allgemeinen Wirtschafts-, Sozial- und Technikgeschichte einzuordnen sind. Vor allem fehlt es gänzlich noch an quantitativ gesicherten Erkenntnissen, wann, wo und wie die neue Tiefkühlkost von den verbrauchenden Haushalten akzeptiert und in die traditionellen Mahlzeitensysteme eingegliedert wurde. Vergleichende Analysen über die räumliche wie zeitliche Diffusion des naturwissenschaftlich-technischen Wissens über die Kältetechnik und die parallelen Entwicklungen im Ausland erscheinen ebenfalls angezeigt.

Wie wir aus überlieferten Abbildungen, Beschreibungen und archäologischen Funden wissen, gehören das heiße und kalte Dörren sowie Räuchern, aber auch das Salzen bzw. Einlegen in Salzlake (Pökeln) zu den ältesten Methoden des Haltbar-

1 Als wissenschaftliche Vorarbeiten für eine umfassende Geschichte der Tiefkühlwirtschaft und Tiefgefriertechnik sind zu nennen: Thévenot, Roger/Fiedler, J.C., Essai pour une histoire du froid artificiel dans les monde, Paris 1978. - Hilck, Erwin und Aufdem Hövel, Rudolf, Jenseits von minus Null. Die Geschichte der deutschen Tiefkühlwirtschaft. Hrsg. vom Deutschen Tiefkühlinstitut e.V., Köln 1979. - Kälte in Wirtschaft und Technik. Aus Anlaß des 10jährigen Jubiläums der Arbeitsgemeinschaft Kälte-Industrie. Beilage zur Zeitschrift „Der Volkswirt”. Wirtschafts- und Finanzzeitung Nr. 37 v. 13. Sept. 1958. - Plank, Rudolf (Hrsg.), Handbuch der Kältetechnik, Berlin-Göttingen-Heidelberg 1954, S. 1-160. - Cummings, Richard Osbor$n e$, The American and His Food, Chicago 1940 (Neudruck New York 1970). - Vgl. Lippmann, Edmund O. von: Zur Geschichte der Kältemischungen, in: Zeitschrift für angewandte Chemie Jg.11 (1898), H.33, S. 739-745.- Wirkner, C.G. von, Geschichte und Theorie der Kälteerzeugung, Hamburg 1897.- Daneben finden sich in Zeitschriften, Firmenfestschriften, Taschenbüchem und Kongreßberichten Notizen zur Entwicklung der Tiefkühlwirtschaft und ihrer Technik. 
machens von Lebensmitteln. ${ }^{2}$ Aus bloßer Beobachtung hatte man offenbar frühzeitig erkannt, daß die hohe Verderblichkeit der meisten Nahrungsstoffe mit ihrem hohen Wassergehalt zusammenhängt, der beim Fleisch je nach Fettgehalt auf $55-$ 75 v.H., bei roher Milch auf rd. 88 v.H. und bei manchen Obst- und Gemüsesorten sogar bis zu 95 v.H. ansteigen kann. Es zeigt sich hier die Wahrheit des alten Alchimistensatzes ,corpora non agunt nisi fluid", d.h. Körper bzw. genauer zersetzende Stoffe wirken nur bei ausreichender Flüssigkeit. ${ }^{3}$ Wie wichtig der Prozeß des Trocknens ist, erlebten die Menschen frühzeitig vor allem beim Getreidekorn, das sich bei nur 13 v.H. Wassergehalt fast unbegrenzt aufbewahren ließ, womit Winter und Notzeiten überbrückt werden konnten. Auch beim Salzen von Fleisch und Fisch wird durch Wasserentzug den pathogenen Fäulnisbakterien der Boden entzogen. Olivenöl, Holz- und Weinessig, hochprozentiger Alkohol sowie Zuckerlösungen und schließlich das Erhitzen der Lebensmittel unter Luftabschluß, von dem französischen Erfinder Denis Papin im späten 17. Jahrhundert erstmals empfohlen, von dem italienischen Gelehrten Lazarro Spalanzi im 18. Jahrhundert erprobt und von dem Leibkoch, Konditor und Likörfabrikanten Nicolas François Appert am Beginn des 19. Jahrhunderts erstmals erfolgreich in die Praxis eingeführt, traten dann als spätere Konservierungsmethoden hinzu. Die ältere Hausväterliteratur, Kochbücher und technische Enzyklopädien unterrichten erstaunlich detailliert, welche Konservierungsarten regional im einzelnen angewandt wurden.

Aus den Schriften antiker Schriftsteller wie Seneca, Petronius, Plinius d. A.. Plutarch, Martial und Juvenal läßt sich erkennen, daß man schon zu ihrer Zei gelegentlich Schnee und Eis zum Kühlen von Fleisch verwandte. Die Verwendung von Schnee und Eis bei den Mahlzeiten der Vornehmen ist seit Beginn der römischen Kaiserzeit vielfach bezeugt. ${ }^{4}$ So begoß man die Hände mit Schneewasser, kühlte die Getränke und besonders den Weinbecher mit Eisstückchen. Die Aufbewahrung des zusammengepreßten Schnees geschah nach Seneca in tiefen Erdlöchern, die man mit Erde, Mist und Baumzweigen zu bedecken pflegte, was nach Athenäus schon Alexander d.Gr. bei Belagerungen angewandt haben soll. Ausführlich wird bei Plutarch die Tatsache erörtert, warum der Schnee in dichte Tücher eingehüllt sich so lange in diesem Zustande erhalten könne. Aus dem bekannten Kochbuch des Marcus Apicius läßt sich entnehmen, daß allerhand gefrorene und mit Schnee bestreute Sülzen bekannt waren. Sein Zeitgenosse Athenäus berichtet von einem fast unglaublichen Kühlluxus: Ließ sich doch der Kaiser Heliogabalus (218-222 n.Chr.) im Sommer neben seiner Villa zum Vergnügen einen Schneeberg anhäufen, während der Usurpator Carinus (um 285 n.Chr.) sogar sein Badewasser aus Schnee und Eis zubereiten ließ. Im alten Mesopotamien und Ägypten, in Indien, China und der peruanischen Inkakultur wurden bestimmte Nahrungsmittel in mit Eis und Schnee gefüllten sowie mit Stroh isolierten Gruben im Sommer vor dem Verderb geschützt. Auch die Hofhaltungen in Damaskus, Bagdad und Kairo zeichneten sich

2 Die älteren Konservierungsmethoden sind gut beschrieben bei Tannahill, Reay, Food in History, New York 1973, S. 210-221. - Vgl. Riddervold, Astri/Ropeid, Andreas(Hrsg.), Food Conservation. Ethnological Studies, London 1988.

3 Ostertag, R. von, Handbuch der Fleischbeschau, 7.-8. Aufl., Stuttgart 1923. Vgl. Rasmussen, L., Die Lebensmittel und ihre Aufbewahrung, Hannover 1931.

4 Die Einzelbelege für die nachfolgende Darstellung bei Lippmann, Geschichte (wie Anm.1). im frühen Mittelalter durch solche luxuriösen Kühlungstechniken aus. So wurde von den arabischen Herrschern gezuckertes Rosenwasser mit Schnee genossen, der aus den Gebirgen des Libanon oder sogar Armeniens herangeschafft wurde. Die Kühlung galt als so wichtig, daß eine besondere Steuer auf das eisgekühlte Wasser erlassen wurde, wie der arabische Geograph Ibn-Haukal (um 900 n.Chr.) erwähnt. Der Kalif Mahdi (775-785) brachte erstmals ganze Schneeladungen auf Kamelen nach Mekka, und einer seiner Nachfolger soll sich gegen die Sommerhitze dadurch geschützt haben, daß er doppelte Wände mit Schnee anfüllen ließ. Auch später haben mittelalterliche Reisende immer wieder über solche Schneetransporte, für die man sogar über bestimmte Relaisstationen verfügte, berichtet.

An diesen uralten Kühltechniken hat sich offenbar jahrhundertelang wenig geändert. Der Schnee- und Eishandel galt in Europa als hoher Luxus, doch war er offenbar selbst an Höfen so gering, daß sich kein Monopol lohnte. Wie eine Abbildung in dem bekannten und von dem Goethefreund Justin Bertuch in Weimar herausgegebenen „Journal des Luxus und der Moden“ 1793 zeigt, gab es zu dieser Zeit auch in Deutschland „Eiskeller”. Dies waren eisgefüllte Gruben mit einem kegelförmigen Strohdach, in denen das Fleisch geschlachteter Tiere aufbewahrt wurde, das hier im Gegensatz zu anderen Konservierungsarten nicht seinen Geschmack und seine Farbe änderte. ${ }^{5}$ Im frühen 19. Jahrhundert bezogen die Neapolitaner regelmäßig im Sommer Eis vom Vesuv, und in Paris sollen jährlich schon etwa 300000 Zentner Natureis verbraucht worden sein, die aus einer $40 \mathrm{Fu}$ tiefen und 132 Fuß großen Eisgrube stammten. Auch von den Alpengletschern wurde Eis rheinabwärts in Zügen verfrachtet.

Anscheinend haben Eiskeller aber zuerst in Nordamerika eine große Verbreitung gefunden, um in dem dortigen feuchtheißen Klima das Fleisch von Haustieren und Wild vor Fäulnis zu bewahren. Später wurden dort dann auch oberirdische Eishäuser gebaut, die nach dem Prinzip der Eiskammern auf Schiffen konstruiert waren. Seit 1805 transportierten nämlich amerikanische Schiffe Natureis zu den Karibikinseln mit ihrer reichen Plantagenwirtschaft, wobei sich nur ein relativ geringer Verlust durch Abschmelzen unterwegs ergab. Schnelle Segel-Clipper brachten natürliches Eis dann sogar nach Rio de Janeiro und Südafrika sowie nach Eröffnung des Suezkanals auch nach Indien.

In den USA befaßten sich im frühen 19. Jahrhundert mehrere Gesellschaften mit dem Schneiden und Lagern von Eisblöcken, die dann hauptsächlich von großen Depots in Boston und Portland ausgeführt wurden. Bei der Gewinnung des natürlichen Eises auf den Großen Seen benutzte man mit Eisenzähnen besetzte Pflüge, spezielle Eisschneider sowie Kratz-, Hobel- und Zerkleinerungswerkzeuge sowie

5 Journal des Luxus und der Moden, Jg. 1793, S. 495. Vgl. zur Verbreitung der Eiskeller im 19. Jahrhundert: Harzer, Friedrich, Vorschriften und Regeln zur Anlegung von Eiskellerm nebst vorausgehender Theorie und Praxis über die Abkühlung der Körper zu wirthschaftlichen und technischen Zwecken. Für herrschaftliche und landwirthschaftliche Haushaltungen, Conditoren, Schlächter etc., Weimar 1853. - Nöthling, Ernst, Die Eiskeller, Eishäuser und Eisschränke, ihre Konstruktion und Benutzung. Für Bautechniker, Brauereibesitzer, Landwirte, Schlächter, Konditoren, Gastwirte usw, 5. umgearb. und verm. Aufl., Weimar 1896 . Swoboda, $K$., Anlegung und Benutzung der Eiskeller, Weimar 1886. 
spiralförmige Förderanlagen. ${ }^{6}$ Als Eis zur Kühlung von Milch und Molkereiprodukten erstmals eingesetzt wurde, nahm die Zahl der ,ice cars" in den Städten sprunghaft zu. Der Umfang der leicht verderblichen Lebensmittel, die mit der Eisenbahn versandt wurden, stieg noch weiter, als ein Erfinder aus Illinois, Parker Earl of Cobden, in den 1840er Jahren eine tragbare Kühlkiste auf den Markt brachte, die sich leicht mit Eis fülen und als Bauchladen herumtragen ließ. Da aber diese Eiskisten auf die Dauer zu klein waren, baute man nun größere „Refrigerators“ mit einer zusätzlichen Ventilation. Seit den 1860er Jahren wurde mit diesen neuen Kühlboxen schon frisches Obst aus dem sonnigen Florida Hunderte von Meilen in die nördlichen Industriegroßstädte geliefert. ${ }^{7} \mathrm{Da}$ aber die Eisenbahnlinien noch nicht überall über durchgehende Strecken verfügten, mußten die natureisgekühlten Lebensmittel unterwegs mehrfach umgeladen werden, was der Qualität abträglich war. Seit 1857 wurde darum versucht, leicht verderbliches frisch geschlachtetes Fleisch, insbesondere teures Wild und Geflügel, vom Ohio Valley nach New York in besondere Kühlwagen der Eisenbahn zu verfrachten. Nachdem das Problem der Vereinheitlichung der Spurweiten gelöst war, konnte die „Star Union Transportation Company" 1865 bereits 30 ,ice boxes on wheels“ anbieten. ${ }^{8}$ Natürlich waren diese Kühlwagen noch recht primitiv: Zwar hatte man die Wände mit Strohmatten isoliert, die Kühlung geschah aber allein durch einen Eiskasten in der Mitte des Waggons, wobei das Schmelzwasser durch Bodenlöcher ablief. J.B. Sutherland aus Detroit ließ sich 1869 das Patent für einen neuen Kühlwagen geben, bei dem mehrere Eiskästen an der Decke angebracht waren und der Waggon eine bessere Luftzirkulation erhielt. Die ,Sutherland cars“ wurden zum Prototyp aller Kühlwagen, die in die urbanen Zentren Frischfleisch transportierten. Die zuerst in Cincinnati, dann in Chicago sich konzentrierende Fleischwarenindustrie wurde nun in den USA zum großen Promotor der Kühlkost. Viele Jahrzehnte waren große Rindviehherden aus dem berühmten ,long drive" von den Prärien des mittleren Westens nach Norden getrieben worden. Von Texas nach Kansas benötigten sie je nach dem örtlichen ,trail“ zwei bis fünf Monate. Durch die Eisenbahnen verkürzte sich nun der Transport des Lebendviehs auf wenige Tage, so daß die Fleischfabriken einen großen Aufschwung nahmen. Das in großen Mengen anfallende Schlachtfleisch mußte nun möglichst schnell über weite Strecken an die amerikanische Ostküste zu den großen Märkten versandt werden.

Schon 1867 ließ der Viehzüchter George Henry Hammond im Bundesstaat Kansas geschlachtete Tiere in Kühlwagen nach Chicago verfrachten und konnte so die dreifache Menge auf der $1000 \mathrm{~km}$ langen Strecke transportieren und alle Konkurrenten unterbieten. ${ }^{9}$ Alle diese Versuche wirkten revolutionierend auf die Viehwirtschaft und Landwirtschaft sowie die Versorgung der großen Städte. Gustavus Swift war der erste große ,meat packer" in Chicago, der viel Kapital in den Bau neuer Kühlwagen und eine möglichst geschlossene Kühlkette steckte. Sein Konkur-

6 Vgl. für das nachfolgende Gideon, Siegfried, Die Herrschaft der Mechanisierung. A.d. Engl., Frankfurt a.M. 1982.

7 Cummings, The American (wie Anm. 1), S. 60ff.

8 Ebd.

9 (Barnett, Corelli u.a.), Vom Faustkeil zum Laserstrahl. Die Erfindungen der Menschheit von A-Z, Stuttgart-Zürich-Wien 1982, S. 167. rent Ogden Armour und alle anderen großen Fleischfabrikanten folgten in den 1870 er Jahren bald nach. ${ }^{10}$ Washington Porter, ebenfalls aus Chicago, ubernahm das neue Kühlwagenprinzip für den Transport von frischem Obst und Gemüse.

Seine umwälzende Neuerung läßt sich hier gut am Beispiel der Banane demonstrieren: 1804 waren erstmals 30 Bündel eßbarer Bananen aus Kuba in New York aufgetaucht. Sie waren in unreifem Zustand gepflückt worden und hatten die Seereise nur dank besonders günstiger Winde und Temperaturen einigermaßen uberstanden. Eigentlich waren sie auch nur zum Verzehr für unterwegs an Bord bestimmt gewesen und ein Verpflegungsrest. 1830 unternahm ein Händler namens Harriet Smith den Versuch eines ersten Bananenimportes, was von dem Autor der Lederstrumpfgeschichten James Fennimore Cooper in einem Bericht erwähnt wurde. Schon ab 1842 eroberten die „,bananas" mit Hilfe der Kühlwagen von der Pazifikküste aus ganz Nordamerika. ${ }^{11}$ Die Abbildung eines Kühlwagens aus dem Jahre 1884 zeigt zwei mit porösem Material verkleidete Kühlräume mit einer Wasserpumpe, die von den Eisenbahnachsen angetrieben wurde. Sie hielt das Gefriergut feucht, während ein Ventilator dafür sorgte, daß ständig Wasser verdunstete und die Kammern mit dem Eis so kalt blieben. Die neuen Kühlwagen können in ihrer Bedeutung für den Beginn des Tiefkühlzeitalters kaum überschätzt werden: Erstmals in der Geschichte wurden frisches Fleisch, Gemüse und Obst aus weit entfernten Erzeugergebieten in die Großstädte gebracht, und dies entlastete die Versorgung durch das Umland. Die immer schneller vor sich gehende Urbanisierung in den USA wäre ohne diese Kühltransporte wohl kaum so schnell möglich gewesen. Die Kühlung von Lebensmitteln mit Hilfe des Natureises blieb im ganzen 19. Jahrhundert ein höchst gewinnbringendes Geschäft, bei dem Tausende von Menschen beschäftigt und Kapital in Millionenhöhe investiert wurde. Auch in Europa blieb die „Eismeierei“ mit ihren Eisgruben und Eishäusern bis in die dreißiger Jahre des 20. Jahrhunderts die vorherrschende Methode zur Kühlung von Lebensmitteln.

So war bereits 1786 in England versucht worden, den kostbaren Lachs von Schottland nach London in Eiskisten zu transportieren. Das notwendige Eis wurde in den zahlreichen schottischen ,lochs" (Seen) gehackt und in feuchten Erdhütten aufbewahrt. Auch die sich ausdehnende Heringsfischerei machte ab 1800 von der Eiskonservierung Gebrauch, so daß ab 1820 mit dem ersten Ausbau des Eisenbahnwesens frischer Seefisch überall ins Landesinnere vordrang. Um 1850 versuchte ein Fischhändler namens Hewett, bereits auf hoher See gesalzenen Hering mit Eis länger haltbar zu machen. Die Fischer aus Grimsly und Hull, die ihr Eis aus Norwegen bezogen, konnten ihre Fangreisen erstmals bis nach Island ausdehnen, und die fischverarbeitende Industrie nahm einen großen Aufschwung. Die Farmer in der Umgebung Londons sahen ein lohnendes neues Geschäft darin, in den Themsemarschen Eisblöcke abzuhacken und zu dem zentralen Fischmarkt Bil-

10 Cummings, The American (wie Anm.1), S.62.- Clemens, Rudolph A., American Livestock and Meat Industrie, New York 1923.- Armour, Ogden, The Packers, the Private Car Lines and the People, Chicago 1906.

11 Root, Waverley and Rochmond, Richard de, Eating in America, New York 1976, S. 150-155. - Levenstein, Harvey A., Revolution at the Table. The Transformation of the American Diet New York-Oxford 1988, S. 30-43. 
lingsgate zu karren. 1902 importierte Großbritannien bereits für 4 Mill. $£$ frischen Seefisch. ${ }^{12}$ Die nun überall entstehenden Imbißstände für ,fish and chips" können als äußerliches Indiz dafür angesehen werden, daß durch die Ausbreitung des Kühlprinzips der frische Seefisch zu einer billigen nationalen Volksspeise wurde.

Eine ähnliche Entwicklung gab es in Deutschland zu beobachten: Die deutsche Seefischerei war lange Jahrhunderte nur als Küstenfischerei betrieben worden und diente der Ernährung der Küstenbewohner. Eine Wende brachten die Jahre nach 1880, als das Eisenbahnnetz praktisch geschlossen war und eine schnelle Beförderung zu den großen inländischen Märkten möglich wurde. Die seit 1886 mit staatlicher Unterstützung ausgebaute deutsche Hochseefischerei trat in ein neues Stadium ein: Die Fischereihäfen wurden ausgebaut und Dampftrawler nach englischem Vorbild in Auftrag gegeben. Neue Fanggebiete, maschinelle Netzwinden, Scherbretter sowie drahtlose Telegraphie steigerten die Fangerträge beträchtlich. Mehrere neue Nutzfischarten, wie z.B. der Rotbarsch, gelangten nun erstmals auf den Markt. Nachdem Dänemark 1854 erstmals eine Fischsendung in Natureispackung auf den Weg gebracht hatte, entstanden nach diesem Beispiel seit 1870 auch in deutschen Großstädten erste Kühlhäuser. Im Winter fuhren deutsche Schiffe nach Norwegen, um in großen Mengen Polareis für diese Kühlhäuser zu holen. Besondere „Fischzüge" von den großen Fischauktionen rollten direkt ins Binnenland. Ab 1890 besaßen alle großen deutschen Seefischereihäfen auch eigene Eiswerke. ${ }^{13}$ Um 1900 gab es allein in London 20 solcher Kühlhäuser mit einer Kapazität für 1,7 Mill. geschlachtete Tierkörper, 1912 waren daraus 29 für 3 Mill. Tierkörper geworden. Entsprechend stieg die Menge des aus Norwegen importierten Natureises seit den 1860er Jahren ständig an: Waren es 1865 erst 38605 t gewesen, so kletterte dieser Import bis 1899 auf rd. 500000 t Natureis..$^{14}$ Aus Platzgründen mußte das Fleisch zusammen mit Obst, Gemüse sowie Butter und Käse gestapelt werden, obwohl alle diese Lebensmittel verschiedene Kühlgrade verlangten. Aber die Betreiber der meist privaten Kühlhäuser mußten mehr Kühlung aufwenden und hatten weniger Gewinn, wenn die Lagerhallen nicht voll ausgenutzt wurden. Die neuen Kühlhäuser, die auch in anderen großen Hafenstädten entstanden, konnten das traditionelle Schwanken der Preise für importiertes Fleisch und Seefisch, das mit der Unregelmäßigkeit der einlaufenden Schiffe zusammenhing, jedoch schon wesentlich herabdrücken.

Wegen der rasch ansteigenden Nachfrage nach Natureis beschäftigten sich nun

12 Burnett, John, Plenty and Want. A Social History of Diet in England from 1815 to the Present Day, London 1966, S. 135

13 Vgl. Rudolph W., Nahrung und Rohstoffe aus dem Meer, Stuttgart 1946, S. 47ff.- Vgl. Treue, Wilhelm, Das Aufkommen der Ernährungsindustrie, in Heischkel-Artelt, Edith (Hrsg.), Ernährung und Ernährungslehre im 19. Jahrhundert, Göttingen 1976, S. 108. - Wiegelmann, Günter und Maus, Annette, Fischversorgung und Fischspeisen im 19. und 20. Jahrhundert: Vernuch einer quantitativen Analyse in: Teuteberg, Hans J. und Wiegelmann, Günhunders. Versuch iner quantitativen Analyse, in: Teuteberg, Hans J. und Wiegelmann, Günter (Hrsg.), Unsere tägliche Kost. Geschichte und regionale Prägung, 2. Aufl., Münster 1986, S. 75-92. Hitzbleck, H., Die Bedeutung des Fisches für die Ernährungswirtschaft Mitteleuropas in vorindustrieller Zeit, Diss. Göttingen 1971. - Stahmer, Max, Fischindustrie und Fischhandel, Hamburg 1913 (2. verb. Aufl. 1943).

14 Perren, Richard, The Meat Trade in Britain, 1840-1914, London- Henley-Boston 1978, S. immer mehr Wissenschaftler mit dem Problem, eine Kühlung der Lebensmittel auf künstlichem Wege zu erreichen. Auch dafür gibt es eine längere Vorgeschichte: Schon in den ersten Hochkulturen der Menschheit hatte man die Erkenntnis gewonnen, daß verdunstendes Wasser kühlt und die Auflösung von Salz diesen Kühlungsprozeß noch verstärkt. So berichtet der griechische Galenos (131-200 n. Chr.), daß man in Ägypten erwärmtes Wasser in Tonschalen gieße, diese nachts auf den Dächern sich abkühlen lasse und dann vor Sonnenaufgang in feuchte Erdgruben bringe und mit feuchten Blättern bedecke. Seit der Mitte des 16. Jahrhunderts finden sich bei Gelehrten wie Blasius Villafranca und Latinus Tancredus sowie dann bei dem Dänen Thomas Bartholinus erste Beschreibungen über die Kühlung von Getränken durch eine Mischung von Wasser oder Schnee mit Salpeter sowie Kochsalz. ${ }^{15}$ Der von 1611-1624 an der Universität Padua lehrende Mediziner Santoro Santorini, der sich unter anderem besonders mit der Ernährung beschäftigte, gab 1626 in seinem Kommentar zu den Schriften des berühmten arabischen Arztes Avicenna (eigentlich Ibn Sina 980-1037) an, daß man zur künstlichen Kälteerzeugung ein Gemenge aus Salpeter und Eis durch eine Mischung von drei Teilen Schnee und einem Teil Kochsalz ersetzen könne, auch in Wasser aufgelöster Salmiak zeige eine ähnliche Reaktion. Dies deutet nochmals auf eine viel ältere Beschäftigung mit diesem Problem im arabischen Mittelalter hin, das seinerseits bekanntlich von den Schriften der Antike zehrte.

Zum eigentlichen Schrittmacher der künstlichen Tiefgefrierung wurden nun die neuen Naturwissenschaften. Ob der englische Naturphilosoph Francis Bacon bereits 1626 versuchte, ein Huhn durch Ausfüllen mit Schnee eine Zeitlang zu konservieren, ist eine bisher unbelegte Nachricht ohne weitere Einzelheiten geblieben. Sicher läßt sich dagegen nachweisen, daß der englische Chemiker Robert Boyle, der erstmals das Gesetz zur Kompression von Gasen gefunden hatte, 1665-1668 Versuchsreihen über solche Kältemischungen veröffentlichte und dabei erwähnte, daß die Vermischung von Schwefelsäure, Salzsäure und besonders Salpetersäure mit Schnee eine künstliche Kälte erzeugt habe. Er erklärte diese Wirkung damit, daß die Salze offenbar den Aggregatzustand von Eis und Schnee verändert hätten. Er hatte richtig erkannt, daß beim Schmelzen bzw. Verdunsten von Eis und Schnee Kälte freigesetzt wird. ${ }^{16}$

15 Villafranca, Blasius, Methodus refrigandi ex vocatu salenitro vinum, aquamque de potus quodiis aliud genus, cui accedunt varia naturalia rerum problemato, non minus iucunda lectu, quam necessaria cognitu, Rom 1550. - Tancredus, Latinus, De fame et siti libri III, physicis ac medicis reconditis controversiis parsim respersi, rerumque varitate omnibus litterarum studiosis perutiles et periocundi, Venedig 1607. - Bartholinus, Thomas, De nivis usu medico observationes variae. Acessit D. Erasmi Bartholini de figura nivis dissertatio cum operum authoris catalogo, Kopenhagen 1661. - Vgl. Beckmann, Johann, Beyträge zur Geschichte der Erfindungen, Theil 4, Leipzig 1799, S. 197.

16 Boyle, Robert, The Works, ed. by Thomas Birch, Bd.2, London 1744, S. 200, S. 266 und S. 300. Vgl. Philosophical Transactions: Giving some Accompt of the Present Undertakings, Studies,
and Labours of the Ingenious in many considerable Parts of the World, vol. I, London 1665, and Labours of the Ingenious in many considerable Parts of the World, vol. I, London 1665,
p. 8-9. (An Experimental History of Cold), pp. 46-52. (A further Experiment of Mr. Boyle's p. 8-9. (An Experimental History of Cold), pp. 46-52. (A further Experiment of Mr. Boyle's
Experimental History of Cold), pp. 344-352. (Promiscuous Inquiries, chiefly about Cold), vol. I, London 1666, pp. 256-262. (A new Frigorifick Experiment shewing, how a considerable degree of Cold may be suddenly produced without the help of Snow, Ice, Haile, Wind, or Niter, and that any time of the year). 
$\mathrm{Zu}$ Beginn des 18. Jahrhunderts hatte man unabhängig davon sich auch mit Kreislaufprozessen der Physik und ihrer praktischen Anwendbarkeit zu beschäftigen begonnen. Lebhaft erörtert wurden alle Bedingungen, unter denen sich Gas in Flüssigkeit und Flüssiges wieder in feste Materie verwandelt. Der Glasgower Universitätsmechaniker James Watt konstruierte bekanntlich damals einen ersten brauchbaren Kondensator, der eine entscheidende Verbesserung der schon bestehenden Dampfmaschine möglich machte. Die nun sich schnell verbreiternden Überlegungen für eine mechanische Kälteerzeugung verfolgten das gleiche Prinzip: Eine Flüssigkeit mit tiefem Siedepunkt wurde verdampft und dann wieder verflüssigt. Beim Verdampfen wurde jedesmal der Umgebung Wärme entzogen oder anders betrachtet Kälte erzeugt. Der englische Physiker Michael Faraday, der erstmals exakt die Verflüssigung von Gasen nachwies, kam bei der Beobachtung verschiedener Gase außerdem zu der grundlegenden Entdeckung, daß Amoniakgas, das er beim Erhitzen in einem U-Rohr erzeugte, sich am anderen Ende wieder zu einer kühlenden Substanz kondensierte. Überläßt man das Amoniak sich selbst, so folgerte er, dann verflüchtigt es sich und erzeugt eine künstliche Kälte. 1788 berichtete der englische Naturwisssenschaftler Charles Blagden, der sich zuvor wie der aus Danzig stammende Physiker Gabriel Fahrenheit mit dem Gefrieren des Quecksilbers beschäftigt hatte, über allerhand Experimente über die Abkühlung des Wassers unter den Gefrierpunkt, wobei er durch verschiedenste Zusätze allerhand Kältemischungen ausprobierte. ${ }^{17}$ Damit waren einige wichtige theoretische Einsichten gewonnen, doch erwies sich die Anwendung in der Praxis als schwierig. Zwar nahmen schon bald danach zwei andere Engländer namens Robert Salmon und William Warres 1819 ein erstes Patent auf eine künstliche Kühlung, und ihr Landsmann John Vallance stellte 1824 erste Versuche an, die Verdunstungskälte zur Herstellung von größeren künstlichen Eismengen zu nutzen, indem er in Schwefelsäure getrocknete und mit einer Luftpumpe stark verdünnte Luft über eine Wasserschicht streichen ließ, aber dies führte noch zu keiner wirtschaftlich rentablen Kühlung.

Sehr viel folgenreicher erwies sich die Konstruktion des ebenfalls aus Glasgow stammenden Maschinendruckers James Harrison, der sich die Beobachtung zunutze machte, daß sich beim Putzen der Maschinen mit Äther (Methylchlorid) diese stark abkühlten. Richtig erkannte er, daß auch hier der Umgebung Wärme entzogen wurde. Er baute, als er 1837 nach Australien ausgewandert war, eine neue Kühlmaschine, bei der Äther mit Pumpen stark komprimiert wurde. Bei verringertem Druck verwandelte sich der Äther in Dampf und erzeugte dabei Kälte. 1851 wurde eine solche Anlage für eine Bierbrauerei in Bendigo im australischen Bundesstaat Victoria erstmals installiert, die zum Prototyp aller nachfolgenden ähnlichen Maschinen wurde. ${ }^{18}$ Als Herausgeber der Zeitung „Melbourne Age“ erkannte Harrison

17 Blagden, Charles, Die Gesetze der Überkaltung und Gefrierpunkterniedrigung. Zwei Abhandlungen (1788). Hg. von A.J. von Oettingen, Leipzig 1894 (=Ostwalds Klassiker der exakten Wissenschaften).

18 Singer, Charles et al.(Hrsg.), A History of Technology, Bd. 5, Oxford 1958, S. 45-46. Eine gute Zusammenfassung aller dieser früheren Versuche zum Bau von Eismaschinen findet sich be Figuier, Louis Guillaume, Les Merveilles d'Industrie, ou Description des Principles Industries Modernes, Bd.3, Paris 1876, S. 591-632. - Vgl. Archer, William Henry, Abstracts of English auch die große Chance, mit Hilfe der künstlichen Kühlung den großen Schaffarmern zu neuem Einkommen zu verhelfen. Hatten sie bis dahin nur die Wolle ausgeführt, so wollte er nun auch das Schaffleisch erstmals auf den europäischen Markt bringen. Er baute in Rodey Point in Victoria hierfür eine spezielle Eisfabrik, konstruierte weitere Eismaschinen und führte 1873 auf einem öffentlichen Bankett Fleisch von Schafen, Rindern, Geflügel sowie Fischen vor, das er sechs Monate eingefroren hatte. Letztlich konnte sich Harrison aber nicht durchsetzen, da es zu dieser Zeit immer noch billiger war, Natureis nach Australien zu importieren. Er war, wie eine genauere Durchmusterung der Patente zeigt, nicht der erste Erfinder, sondern hatte wahrscheinlich die „Äthereismaschine“ des in England lebenden Amerikaners Jacob Perkins weiter entwickelt, der bereits 1834 eine ähnliche Anlage gebaut hatte. Neben diesem gab es noch weitere Versuche zum Bau von ,air compression machines", die aber offenbar alle nicht zu praktischen Zwecken eingesetzt wurden und ihrer Zeit vorauseilten.

Den eigentlichen Durchbruch erzielte erst der französische Zivilingenieur Fernand Philippe Edouard Carré, der 1857 eine Ammoniak-Absorptionsmaschine konstruierte, in der das Wasser durch rasche Verdunstung von kondensiertem Ammoniak zum Gefrieren gebracht wurde. Seine Eismaschine konnte bereits künstliches Eis in mehreren tausend Pfund herstellen und war daher auf der Pariser Welt-Industrieausstellung 1862 eine kleine Sensation. Carré baute auch einen ersten Kühlschrank für die häusliche Küche. Sein ,appareil refrigérant pour la production de la glace" benutzte ebenfalls Ammoniak als Gefriermittel. Es handelte sich hierbei um einen tragbaren mit Ammoniak gefüllten Kessel, einen Gefrierbehälter sowie ein Reservoir. Dabei brauchte man etwa zwei Stunden, um $1 \mathrm{~kg}$ Kunsteis zu erzeugen. Kein Wunder, daß diese „Eismaschine“ für normale Haushalte schon wegen des Anschaffungspreises nicht in Frage kam. Immer neue „Refrigeratoren“ wurden seit dieser Zeit konstruiert, wobei man meistens wie Harrison auf der Perkins'schen „Äthereismaschine“ sowie auf den seit 1850 bekannten Kaltluftmaschinen aufzubauen versuchte. ${ }^{19}$

1861 wurde in Darlington Harbour bei Sidney durch Thomas Sutcliffe Mort eine erste Fabrik für Gefrierfleisch errichtet, nachdem er mit dem französischen Ingenieur E.D. Nicolle zahlreiche Versuche angestellt hatte. Mort gründete die ,New South Wales Fresh Food and Ice Company", die wie Carré das Ammoniak-Kompressions-Prinzip anwandte. 1875 folgte der Bau eines Schlachthauses mit Kühlräumen. Beide Unternehmen arbeiteten sofort zwar technisch einwandfrei, mußten aber ebenso wie Harrison später aus wirtschaftlichen Gründen Konkurs anmelden. Offenbar war es noch um 1870 nur möglich, mit Salz-Eismischungen leicht gekühltes Rindfleisch von den USA nach England zu verschiffen. 1877 transportierte das französische Kühlschiff „Le Frigorifique“ erstmals mit Hilfe einer mechanischen Kühlung probeweise Fleisch von Rouen nach Buenos Aires..$^{20}$ Der

and Colonials Patent Specifications Relating to the Preservation of Food etc. Compiled from original documents, of their printed copies, logded to the Patent Office, attached to the Registrar-General's Department, Melbourne 1870.

19 Feldhaus, Franz Maria, Die Technik. Ein Lexikon der Vorzeit, der geschichtlichen Zeit und der Naturvölker (1914), 2. Aufl., München 1970, S. 549.

20 Tellier, Charles, Historie d'une Invention Moderne: le Frigorifique, Paris 1910. 
französische Ingenieur Charles Tellier, der auf den Ideen von Carré aufbaute, kühlte Wasser in großen Tanks, das durch Kühlrohre durch den Laderaum gepumpt wurde. Auf der Rückfahrt 1877, die 104 Tage dauerte, war aber das argentinische Fleisch verdorben, so daß er das Problem noch nicht gelöst hatte. Eine erfolgreiche Fahrt mit 80 t gefrorenem Hammelfleisch von Buenos Aires nach Le Havre schaffte dann im gleichen Jahr das Schiff „Paraguay”, das wiederum eine Ammoniak-Absorptionsmaschine nach dem Vorbild von Carré an Bord hatte. Obwohl die Schiffsreise wegen einer Schiffskollision sechs Monate dauerte, besaß das bei minus 30 Grad Celsius eingefrorene Hammelfleisch bei der Ankunft eine ausgezeichnete Qualität.

Dieser Erfolg stimulierte die Anstrengungen in Australien, mit den Versuchen zur Versendung von Gefrierfleisch fortzufahren. 1879 segelte die „Strathleven" von Plymouth nach Australien und kam am 2. Februar 1880 mit 40 t Rind- und Hammelfleisch zurück, das sich weder im Aussehen noch im Geschmack von frischer Ware des nächsten Fleischladens unterschied. Um 1880 begann auch Neuseeland, wo sich die Schafherden von 23000 im Jahre 1851 auf 11 Millionen Tiere vermehrt hatten, auf einer Reise von 98 Tagen Gefrierfleisch nach England zu verfrachten. Die Einfuhr wuchs von 2 Mill. Tierkörpern 1892 auf 4 Mill. zehn Jahre später. Um 1900 versandte Argentinien 2 Mill. Lamm- und Hammelkörper und $412262 \mathrm{t}$ gefrorenes Rindfleisch. Nachdem die USA wegen rasch steigender Bevölkerungszahlen immer weniger Fleisch liefern konnten, wurden Australien, Neuseeland und Argentinien nun zu den großen Gefrierfleischexporteuren der Welt. ${ }^{21}$ Buenos Aires ,klirrte vor Reichtum“ und stieg zur sechstgrößten Weltstadt auf.

Aber auch in Europa gingen zu dieser Zeit die Versuche weiter. Gegen Ende der 1850er Jahre setzte sich auch in den Ländern, wo man an sich genügend Natureis gewinnen konnte, immer mehr die Erkenntnis durch, daß es ökonomischer sei, zu einer künstlichen Kälteerzeugung überzugehen. Die Physik hatte inzwischen gelehrt, daß es drei Wege gibt, eine Temperatur künstlich zu erniedrigen und durch Übertragung auf Wasser Eis zu erzeugen: 1. durch die Auflösung oder Verflüssigung fester Körper (z.B. von Salzen), 2. durch künstliche Verdampfung von Flüssigkeiten sowie 3. durch die Ausdehnung von Gasen. Die erste Methode schien für die nicht kontinuierliche Herstellung von Eis in kleinen Mengen, die beiden anderen Methoden zur kontinuierlichen Fabrikation von Eis mit Hilfe komplizierter Maschinen am besten anwendbar zu sein. Verständlicherweise wandte man sich zunächst dem ersten und billigeren Verfahren zu. So führte der Italiener Toselli am 10. August 1868 der französischen Akademie der Wissenschaften einen Eisblock von $20 \mathrm{~cm}$ Breite und $50 \mathrm{~cm}$ Höhe vor, den er in nur 12 Minuten mit einem von ihm erfundenen Eiserzeugungsapparat hergestellt hatte. Es handelte sich um eine einfache Blechbüchse, in die eine konisch geformte Blechröhre eingehängt wurde. Eine fest schließende Gummidichtung verband beide am Rand. Nachdem man das Gefriergut in die innere Röhre getan hatte, wurde die Büchse mit Salz und Wasser als Kältemischung gefüllt. Nach Verschluß wurde das äußere Gefäß mit einem Tuchmantel umgeben und dann auf dem Tisch kräftig hin und her gerollt. Bei dieser Kältemischung, der dann auch später zur Hälfte salpetersaurer Ammoniak zugesetzt wurde, konnte eine Temperatur von 2-3 Grad Celsius unter Null künstlich erzeugt werden.

21 Singer, History (wie Anm. 18). - Vgl. Perren, Meat trade (wie Anm. 14).
Tosellis Eismaschine, die als "glacière italienne roulante" in den Handel gebracht wurde, fortesterten Nachahmungen an. So stellte der Professor H. Meidinger, der Direktor der „Großherzoglich-Badischen Landes-Gewerbehalle ${ }^{6}$ in Karlsruhe (dem Vorläufer der späteren Technischen Hochschule), einen ähnlichen Apparat vor. ${ }^{22}$ Sein Kälteapparat wurde 1873 auf der Weltindustrieausstellung in Wien vorgeführt und war wie der von Toselli vor allem für die kleingewerblich-häusliche Eisherstellung im Kleinen gedacht, weshalb sich Konditoren dafür interessierten. Die Firma C. Beuthenmüller in Bretten entschlo $ß$ sich daraufhin, solche rotierenden neuen Eismaschinen in größerer Zahl herzustellen. Aber offenbar haben diese Apparate dann doch keine große Verbreitung finden können. Das Verhältnis der aufgewandten Salzmischung zur erzielten Eismenge war zu ungünstig, und das Eis hatte eine zu hohe Temperatur. Hatte man besonders im Sommer kein wirklich kaltes Wasser, so war das Ergebnis noch unsicherer. Ein Abdampfen der Salzlösung zur Wiedergewinnung des Salzes konnte in der häuslichen Küche oder kleinen Werkstatt nicht vorgenommen werden und verteuerte den Einsatz. Physikalisch richtige Erkenntnisse konnten so noch nicht in eine wirtschaftlich verwertbare Form umgesetzt werden.

Ein großer Erfolg in der Kühltechnik gelang dann dem Ingenieur Carl Linde (1842-1934). ${ }^{23}$ Der Altmeister der deutschen Kältetechnik, Sohn eines in ärmlichen Verhältnissen aufgewachsenen und dann mit einer Kaufmannstochter verheirateten protestantischen Pfarrers in Franken, hatte zuerst auf dem Polytechnikum Zürich und dann bei der Königl. Preußischen Gewerbeakademie, dem Vorläufer der heutigen Technischen Universität Berlin, vor allem bei Franz Reuleaux studiert, bei den gerade entstandenen Lokomotivfabriken von August Borsig in Berlin und Georg Krauss in München seine Lehr- und Gesellenjahre erfolgreich abgeleistet, um schon mit 26 Jahren als außerordentlicher Professor für Maschinenlehre an das neugeschaffene Polytechnikum München berufen zu werden. Entscheidend für seine Lebensarbeit wurde seine 1870 vorgelegte Schrift „Wärmeentziehung bei niedrigen Temperaturen durch mechanische Mittel”. 1871 erschien seine ebenfalls epochemachende Abhandlung über ,Verbesserte Eis- und Kühlmaschinen”, die Bierbrauer in der Stadt aufhorchen ließ. Der bekannte Brauereibesitzer Georg Sedlmayr war sofort bereit, eine erste solche Maschine auf seine Kosten konstruieren zu lassen und stellte Räume für Versuche bereit.

Linde kam es im Gegensatz zu anderen Erfindern von Anfang an nicht nur darauf an, das Natureis zu ersetzen, sondern auch Mittel zur weiteren Verwendung und Übertragung der künstlichen Kälte zu finden. 1873 stellte er auf der Wiener Weltausstellung bei einem Internationalen Brauereikongreß sein neues Programm

22 Vgl. Toselli's Verfahren, um Eis von bedeutender Dicke zu fabricieren (Aus Les Mondes, tome XVIII, p.627, August 1868), in: Dingler's polytechnisches Journal Bd.190 (1868), S. 26.Meidinger, [H.],Eismaschine zur Bereitung von Gefrorenem, in: ebd., Bd.204(1872), S.409-414.Ders., Die Fortschritte in der Erzeugung von künstlichem Eis, in: ebd. Bd.217(1875), S. 471-478.

23 Matschoß, Conrad, Große Ingenieure. 4. verb. Aufl., bearb. von Friedrich Hasler, München 1954, S. 360-366. - Linde, Carl von, Aus meinem Leben und meiner Arbeit. Erinnerungen des Pioniers der Kältetechnik (1916). Nachdruck mit 36 Bildtafeln und einem Anhange. Ausgewählte Briefe C. Lindes zwischen 1861 und 1910, Düsseldorf 1984. 
vor. Sein Kerngedanke war, die Kältemaschinen in die bereits hochentwickelte Natureistechnik einzugliedern, aber Flüssigkeiten und Lufträume nun erstmals unmittelbar zu verbinden, um die Kosten für die Kühlung drastisch zu senken. Die bis dahin noch weit billigere Natureisherstellung sollte damit als Konkurrent verdrängt und der künstlichen Kälte zur breiteren Anwendung verholfen werden. Damit erregte Linde international großes Aufsehen. 1875 konstruierte er eine neue Ammoniak-Eismaschine, bei der die Dämpfe des in einem Röhrenapparat (Verdampfer) befindlichen flüssigen Ammoniaks von einer Pumpe angesaugt und dann dem Kondensator zugeführt wurden, wo sie unter Mitwirkung des in der Rohrspirale umfließenden Kühlwassers sich zur Flüssigkeit wieder verdichteten, um dann wieder in den Verdampfer zurückzukehren. Auf den Erfahrungen von Kühlanlagen bei Brauereien und Schlachthöfen aufbauend konstruierte er auch ein handliches Haushaltsgerät, das im wesentlichen schon unseren heutigen Kompressorkühlschränken entsprach. Lindes neue Kältemaschine, die im Vergleich mit allen anderen einen sehr viel höheren Wirkungsgrad hatte, hat übrigens zwei weniger vollkommene Vorläufer gehabt, von denen einer zunächst noch wie bei Perkins und Harrison mit Methyläther als Arbeitsflüssigkeit betrieben wurde.

Nachdem zunächst die Maschinenfabrik Augsburg und die Gebrüder Sulzer in Winterthur seine Kältemaschinen gebaut und in ganz Europa vertrieben hatten, begründete er 1879 in Wiesbaden seine eigene Firma, die in einem Zimmer und mit einem Zeichner begann. Seine wichtigsten Kunden waren verständlicherweise zunächst die Bierbrauereien, wo er die gesamte Kältetechnik auf einen neuen Stand zu bringen versuchte. Dann wandte er sich den Fleischkühlanlagen und Kühlhäusern, dem Bau ganzer Eisfabriken, chemischer Unternehmen und Molkereien sowie den Gasverflüssigungsanlagen und den Kühlschränken für den Haushalt zu. Aus Prospekten, Geschäftsberichten, Firmenfestschriften und seinen Memoiren läßt sich das Produktionsprogramm und Wachstum seines Unternehmens, das in der Hauptsache ein „Technisches Central-Bureau“ war, recht gut rekonstruieren. Hatte die „Gesellschaft für Linde's Eismaschinen“ 1877 erst zehn Maschinen im Jahr ausgeliefert, so waren es 1896 schon 429 . Zu dieser Zeit gab es 2756 Kältemaschinen in 1659 gewerblichen Unternehmen, davon 900 in Bierbrauereien, 297 in Fleischfabriken, 122 in Eisfabriken, 109 auf Seeschiffen und 80 in Molkereien. Die meisten seiner Maschinen waren im Deutschen Reich, England und den USA sowie Österreich-Ungarn aufgestellt, doch gab es auch in anderen Ländern Kunden, selbst in Südamerika, China und Ägypten. ${ }^{24}$ Der Durchbruch zum unentbehrlichen Haushaltsgerät wurde erst erreicht, als dieses einen elektrischen Antrieb erhielt. 1910 waren in den USA etwa 200 Haushalte mit Kühlschränken ausgerüstet, zwei Jahre später bot die „Allgemeine Electricitätsgesellschaft“ in Berlin das erste Modell seiner „automatischen Eis- und Kühlmaschine für den Haushalt" an, die freilich noch 1500 Goldmark kostete. Linde hat sich dann mit 49 Jahren ganz auf seine Versuche zur Erforschung von tiefen Temperaturen in seine Kälteversuchsstation an der Universität München zurückgezogen, ohne sich aber ganz von seinen geschäft-

24 Vgl.Linde, Leben (wie Anm. 23). Vgl. Gesellschaft für Lindes Eismaschinen Wiesbaden (Prospekt), Wiesbaden o.J. (1896), Gesellschaft für Lindes Eismaschinen Wiesbaden (Hrsg.), Künstliche Kälte in Fleischereien, Delikatessen-, Wild- und Geflügelhandlungen 0.0 ., [Wiesbaden] o.J. [1929]. lichen Unternehmungen lösen zu können. 1895 gelang Linde aufbauend auf Entdeckungen von William Thompson und James Prescott Joule erstmals die Verflüssigung der Luft, was eine neue Physik der tiefen Temperaturen und erste Untersuchungen auf dem Gebiet mit hohem Vakuum bedeutete.

Neben Carl Linde, der wegen seiner Verdienste vom Kaiser geadelt wurde, ist unbedingt noch eines anderen Pioniers aus der Frühzeit der Tiefkühlwirtschaft zu gedenken, nämlich des dänischen Fischhändlers A.J.A. Ottesen (1860-1936), der eine künstliche Tiefgefrierung des Fisches erstmals einleitete. In dem stark auf Agrarexport und Fischerei ausgerichteten Dänemark hatte man die vielfältigen Versuche zur Kältetechnik aufmerksam im späten 19. Jahrhundert verfolgt, wie die Versuche zur Verbesserung der Eismeierei auf der Landbauschule Kopenhagen zeigen. ${ }^{25}$ Seit der Erfindung einer rentablen Kältekompressionsmaschine war es Ottesen klar, daß man kein Eis mehr brauchte, sondern nur noch eine Salzlösung zu kühlen hatte, um den hochverderblichen Fisch zu schützen. Eine brauchbare Lösung scheiterte aber zunächst immer wieder daran, daß der Frischfisch beim. Eintauchen in ein Solebad soviel Salz in sich aufnahm, daß er nicht mehr genießbar war. Der dänische Fischexporteur fand nach Versuchen nun heraus, daß jede Kochsalzlösung einen ihrem jeweiligen Konzentrationsgrad entsprechenden Gefrierpunkt besitzt, bei dem dann nicht mehr das Salz, sondern das Eis ausgeschieden wird. Ein in diese Lösung eingetauchter Fisch nahm dann nicht mehr Salz, sondern die Kälte auf. ${ }^{26}$ Ottesen wählte einen Salzgehalt von 28,9\%, der bei -21,2 Grad Celsius gefriert. Damit konnten nun Fische je nach Dicke in 1-3 Stunden auf rund minus 20 Grad eingefroren und fast unbegrenzt haltbar gemacht werden. 1912 baute die Kältemaschinenfabrik Sabroe den ersten Apparat zum Tiefgefrieren, und 1915 nahmen entsprechende Industrieanlagen in Esbjerg ihre Produktion auf. Die weitere Entwicklung der deutschen Tiefkühlwirtschaft konnte sich dann auf diesen Grundlagen rasch weiter entfalten.

Auf dem Ottesenschen Patent von 1911 aufbauend legte zunächst der an der Technischen Hochschule Danzig lehrende Professor für Thermodynamik Rudolf Plank 1915/16 seine wichtige Denkschrift „Die Konservierung von Fischen durch das Gefrierverfahren" vor. Ihm und seinen Mitarbeitern war erstmals der Nachweis gelungen, daß schockartig eingesetzte tiefe Temperaturen das Gefriergut am besten erhalten und das Zell- bzw. Muskelgewebe am wenigsten verändern. Der Fischereiexperte Walter Schlienz, vom preußischen Landwirtschaftsministerium in die Geschäftsführung des 1896 gegründeten Unternehmens NORDSEE übergewechselt, wurde zum eifrigsten Fürsprecher eines Tiefgefrierverfahrens und war maßgeblich an der Gründung der Kühlfisch-Aktiengesellschaft, dem ersten verarbeitenden deutschen Tiefkühlunternehmen, 1925 aktiv beteiligt. Die Großproduktion am Fließband, die maschinelle Fischfiletierung und die ersten Fischbratküchen, aber auch die Ausrüstung eines ersten Tiefkühlschiffes, das den Fisch gleich nach dem Fang filetiert und tiefgefriert, und die Gründung der „Gefriertechnischen Gesell-

25 Hilck-Auf dem Hövel, Jenseits von minus Null (wie Anm.1), S.11-18. - Fjord, U.J., Bericht über den Versuch auf dem Gebiet der Eismeierei, ausgeführt auf der Landbauschule zu Kopenhagen und auf den Gütern Durupgaard und Gjedfergaard. A.d. Dänischen von E. Boysen, Hildesheim-Danzig 1877.

26 Hilck-Auf dem Hövel, Jenseits von minus Null (wie Anm.1), S.14. 
schaft deutscher Hochseefischereien" sind ihm mitzuverdanken. ${ }^{27}$

Der amerikanische Fischereibiologe Clarence Birdseye (1886-1956), ein Altersgenosse von Rudolf Plank, stellte in den zwanziger Jahren als Weiterentwicklung des Ottesenschen Sole-Gefrierens seinen neuen Plattenfroster vor, der das Gefriergut zwischen zwei Metallbändern hindurchführte, die von außen mit tiefgekühlter Sole besprüht wurden. ${ }^{28}$ Birdseye stellte auch erste Versuche zum Einfrieren mit kartonverpacktem Gemüse an und machte die amerikanische Eiskremtruhe zum ersten praktischen Kühlmöbel, in dem der Einzelhändler tiefgefrorene Haushaltspackungen anbieten konnte. Seit der Mitte der dreißiger Jahre war der Durchbruch geschafft, und "Quick Frozen Food" feierte in den USA seine ersten triumphalen Erfolge. Die Gesamtproduktion war dort schon auf $170000 \mathrm{t}$ im Jahr gestiegen, wobei Obst und Gemüse gefolgt vom Fisch an der Spitze standen.

In Deutschland brachten der „Vierjahresplan“ ab 1936 und der 2. Weltkrieg eine unerwartete Förderung der Tiefkühltechnik, da das NS-Regime durch eine vermehrte Fischkonservierung die gefürchtete Eiweißlücke in der Volksernährung schließen und Hungersnöte wie im 1. Weltkrieg vermeiden wollte. Die industrielle Herstellung von Eiskrem und kartonverpackten Fischfilets, die Tiefkühlung von Gemüse und Obst in Zusammenarbeit mit der Konservenindustrie, die Konstruktion von Gefrieranhängern bei Lastwagen und Fabrikschiffen sowie die Gründung von zahlreichen Unternehmen und Fachverbänden sind weitere Folgen dieser massiven staatlichen Förderung gewesen. Der weitere Aufschwung der deutschen Tiefkühlindustrie nach $1945 \mathrm{kann}$ als eine logische Fortsetzung aller dieser Initiativen und Aktivitäten bezeichnet werden. Da dieser jüngste Zeitabschnitt der letzten 50 Jahre der Entwicklung der Tiefkühlkost relativ gut bekannt ist, scheint es unnötig, bekannte Namen und Produkte hier nochmals aufzuführen. Stattdessen soll versucht werden, am Schluß die gesamte Entwicklung in ein paar allgemeinere Thesen zu fassen:

1. Die Bemühungen, die Kälte zur Konservierung leicht verderblicher Nahrungsmittel einzusetzen, ist keineswegs neu, sondern gehört zu den frühesten zivilisatorischen Betätigungen der Menschheit in allen Hochkulturen und ist als ein ubiquitäres Phänomen anzusehen. Natureis, Schnee und Wasserverdunstung wurden jahrtausendelang rein empirisch aber nur im sehr begrenzten Rahmen genutzt, bis die seit dem 17. Jahrhundert entstehenden modernen Naturwissenschaften erstmals eine theoretische Durchdringung dieser Vorgänge anbahnten. Vor allem haben die Chemiker und Physiker Robert Boyle, Joseph Priestley und Michael Faraday neben James Prescott Joule und Lord William Kelvin durch die Formulierung von Naturgesetzen der Kältetechnik Wege zur rationellen Verbesserung gewiesen.

27 Plank, Rudolf, Ehrenbaum, E., Reuter, K., Die Konservierung von Fischen durch Gefrierverfahren. In: Abhandlungen zur Volksernährung, Berlin 1915. - Kallert, Eduard und Plank, Rudolf, Über die Behandlung und Verarbeitung von gefrorenem Schweinefleisch. In: Abhandlungen zur Volksernährung, H.1, Berlin 1915. - Plank, Rudolf, Die Frischerhaltung von Lebensmitteln durch Kälte. In: Abhandlungen und Berichte des Deutschen Museums, H.12, München 1940. - Ders., Haushalts-Kältemaschinen, Berlin 1928.

28 Ders., Amerikanische Kältetechnik, Berlin 1929. - Ders., Versuche zur Kaltlagerung von Obst und Gemüse, in: Zeitschrift für die gesamte Kälteindustrie, Beiheft R.3. - Ders., Vorkühlungsanlagen für Obst, in: Zeitschrift für die gesamte Kälteindustrie, Beiheft 3,8 (1937).
2. Vor dem eigentlichen Beginn des Zeitalters der modernen Tiefkühlkost gab es zunächst eine breite Anwendung der Natureiskühlung auf gewerblicher wie privater Grundlage, wobei schon wertvolle Erkenntnisse für die breitere praktische Anwendung des Kühlprozesses gewonnen wurden. Insbesondere hat in den USA die Einführung von Kühlwagen auf den Eisenbahnen und Eiskammern auf Schiffen die Nahrungsversorgung der Städte revolutionär verbessert und die Viehwirtschaft in ungeahntem Maß intensiviert. Die Versorgung mit frischem Obst, Gemüse und Milch, die früher mengenmäßig nur eine geringe Rolle spielte, wurde dadurch erstmals entscheidend verbessert. Aber auch in Deutschland gab es einen ungeheuren Aufschwung der „Eismeierei”, die die Hauswirtschaft und die Mahlzeiten umgestaltete. Die Abhängigkeiten der Lebensmittelversorgung von den Ernte- und Fangterminen wurden damit vermindert, und die großen Preisausschläge auf den Lebensmittelmärkten sind dadurch abgeschwächt worden. Die Versorgung der städtischen Märkte wurde auch dadurch verbessert, daß der frische Seefisch erstmals ins Landesinnere vordrang und die einfache Volkskost spürbar erweiterte. Der Bau von großen Kühlhäusern war in allen Großstädten zu beobachten, wodurch das Lebensmittelangebot stark erweitert und hygienisch verbessert wurde. Bei der Natureisverwendung hatte es sich stets nur um eine Kühllagerung etwa zwischen - 0,5 Grad Celsius und + 5 Grad Celsius ohne Abtötung des Zellgewebes gehandelt.

3. Der sprunghafte Anstieg der Nachfrage nach Natureis mit rasch steigenden Preisen, die Unvollkommenheiten einer Lebensmittelkühlung auf längeren Schiffsreisen, die Kontamination des Natureises und seine schwierigen Transportprobleme müssen als die wichtigsten Ursachen angesehen werden, nach mechanischen Gefriermethoden Ausschau zu halten. Die Entwicklung neuer "Kältemaschinen", die unmittelbar an die physikalisch-chemischen Erkenntnisse des 18. Jahrhunderts anknüpften, waren dadurch im 19. Jahrhundert gekennzeichnet, daß sich drei Verfahren konkurrierend gegenüberstanden:29

- Das Kompressionssystem mit dem wiederholten Kreislauf von Verdichtung, Verflüssigung, Ausdehnung und Verdampfung.

- Das Absorptionssystem, das mit Hilfe von Wärme einen der Verdichtung ähnelnden Vorgang zustande brachte und mit Kälte einen dem Ansaugen entsprechenden.

- Das Luftsystem, bei dem gekühlte komprimierte Luft einen Motor antrieb oder die Luft ausdehnte und kühlte und diese Luft dann zum Gebrauch in Kühlräume leitete.

Bei dieser Tiefgefrierung zwischen -12 und -30 Grad Celsius wurden die Schutzmaßnahmen des lebenden Organismus nun im Gegensatz zur Kühlung ausgeschaltet.

Die Absorptions- und Kaltluftmaschinen haben um 1860 zunächst eine industrielle Bedeutung erlangt, dann aber setzten sich die Kaltdampfanlagen immer mehr durch, wobei Carl Linde ähnlich wie David Boyle in den USA die wirtschaftlich rentabelsten und technisch sinnvollsten Verfahren damals einsetzte. Später schoben sich aber die Absorptionsmaschinen wieder stärker in den Vordergrund, da sie die Abwärme von Großanlagen nutzten und fast ohne Ver-

29 Reader, W., Birds Eye - The Early Years, Walton-on-Thames, Surrey 1963. 
schleißteile gebaut werden konnten. Auch die Kühlschränke im Haushalt wurden vor allem nach dem Absorptionsprinzip gebaut. Damit gehörte aber das bewährte Kaltluftprinzip keineswegs der Vergangenheit an und wurde nun für andere Zwecke benutzt.

4. Zunächst hatte man nur an Kühlgut gedacht, nicht aber an seinen Transport zum verbrauchenden Haushalt. Erst im 20. Jahrhundert sind als spätere Stufen der Entwicklung verschiedene konsumfreundliche Verpackungsformen in den Prozeß des Tiefgefrierens einbezogen worden. Damit konnte die Kühleiskette immer mehr geschlossen, der Absatz durch Werbung zugleich gesteigert und verschiedene Dienstleistungen des Haushalts in die angebotene Ware einbezogen werden.

5. Leider ist über die Entwicklung und Differenzierung des Lebensmitteleinzelhandels mit seinen Umsätzen an Tiefkühlkost sowie die Motivationen der kaufenden Hausfrauen sowie den tatsächlichen häuslichen Konsum nur wenig bekannt. Bis jetzt gibt es nur eine vage Geschichte der Produktion, aber überhaupt keine der Konsumtion. So stehen bis jetzt nur wenige Daten zur Verfügung, die lediglich die Oberfläche des Verbrauches fragmentarisch erhellen. ${ }^{30}$ So besaßen 1960 erst 3 v.H. aller bundesdeutschen Haushalte ein Tiefkühlgerät, 1969 immerhin schon 18 v.H., die aber fast ausschließlich in ländlichen Haushalten standen. 1965 waren erst 52,6 v.H. der Haushalte mit einem einfachen Kühlschrank ausgestattet, 1969 aber schon 85 v.H., von denen aber 1970 erst 10 v.H. ein Drei-Sterne-Fach zum längeren Aufbewahren von Tiefkühlkost besaßen. Der Konsum von Tiefkühlkost stieg zwischen den Jahren 1960 und 1970 von $22100 t$ auf $218992 \mathrm{t}$, was also eine Verzehnfachung bedeutete. Durch die veränderten Handelsformen, insbesondere die tiefgefrorene Fertigkost, die Modernisierung der Groß- und Privatküchen und den weiter angestiegenen Lebensstandard dürfte die Tiefkühlkost ihren Aufschwung in den letzten zwanzig Jahren weiter fortgesetzt haben. Die Jahrzehnte um 1860 wie 1960 scheinen die beiden entscheidenden Zäsuren für die Geschichte der Kühlkost und des Tiefgefrierens gewesen zu sein. Aber erst genauere vergleichende Studien könnten endgültig klären, ob diese Periodisierung richtig ist.

\section{Summary}

The technique of freezing food is one of the great and lasting innovations of the modern industrial age. Methods of stockpiling and the culture of human nourishment were enlarged and revolutionized. Dehydration, salting and the use of snow and ice were the earliest methods of conservation in ancient times. Ice cellars, which were mainly used in North America since the 18th century, were supplied with ice

30 Neuloh, Otto/Teuteberg, Hans J., Ernährungsfehlverhalten im Wohlstand. Ergebnisse einer empirisch-soziologischen Untersuchung in heutigen Familienhaushalten, Paderborn 1979, S. 138-142. - Nisi, Ruth, Tiefkühlkost stark gefragt. In: Landfrau heute. Mitteilungen der Deutschen Landwirtschaftsgesellschaft, Jg.32(1973), S. 917-918 - Institut für Demoskopie Allensbach (Hrsg.), Lebensmittel aus Dosen und Gefriertruhe, (Pressedienst) Allensbach/ Bodensee, August 1966. - Mitteilungen des Deutschen Tiefkühlinstitus e.V. Köln. blocks of the Great Lakes. In the 19th century, important innovational steps in the USA were the conservation of dairy products in so-called "ice cars" and the development of a cooling system in railway-cars, which was of utmost importance, because it offered food industries new markets.

Since ancient times, researchers have been occupied with the problem of artifical refrigeration and big progress has been made by modern natural sciences since the 18 th century. James Watt constructed the first efficiently working condensator; in the 19th century, the principle of ammoniac-compression was developed and refrigerators were produced. They were the basis for the first factories for frozen food.

In the 1870s, the developments of the engineer Carl Linde marked an epoch. With the construction of a mechanical evaporator he presented a cooling system which was more practicable and in the long run even cheaper than natural ice.

Anschrift des Autors: Prof. Dr. H. J. Teuteberg, Westfälische Wilhelms-Universität Münster Historisches Seminar, Domplatz 20-22, 4400 Münster 